A high-throughput metabolomics method to predict high

concentration cytotoxicity of drugs from low concentration profiles

Journal Article

Author(s):

Heux, Stéphanie; Fuchs, Thomas J.; Buhmann, Joachim M.; Zamboni, Nicola (1); Sauer, Uwe

Publication date:

2012-06

Permanent link:

https://doi.org/10.3929/ethz-b-000048939

Rights / license:

In Copyright - Non-Commercial Use Permitted

Originally published in:

Metabolomics 8(3), https://doi.org/10.1007/s11306-011-0386-0 


\title{
A high-throughput metabolomics method to predict high concentration cytotoxicity of drugs from low concentration profiles
}

\author{
Stéphanie Heux · Thomas J. Fuchs • \\ Joachim Buhmann • Nicola Zamboni • \\ Uwe Sauer
}

Received: 29 September 2011 / Accepted: 16 November 2011/Published online: 3 December 2011 (C) Springer Science+Business Media, LLC 2011

\begin{abstract}
A major source of drug attrition in pharmacological development is drug toxicity, which eventually manifests itself in detrimental physiological effects. These effects can be assessed in large sample cohorts, but generating rich sets of output variables that are necessary to predict toxicity from lower drug dosages is problematic. Currently the throughput of methods that enable multiparametric cellular readouts over many drugs and large ranges of concentrations is limited. Since metabolism is at the core of drug toxicity, we develop here a highthroughput intracellular metabolomics platform for relative measurement of 50-100 targeted metabolites by flow injection-tandem mass spectrometry. Specifically we focused on central metabolism of the yeast Saccharomyces cerevisiae because potential cytotoxic effects of drugs can be expected to affect this ubiquitous core network. By machine learning based on intracellular metabolite responses to 41 drugs that were administered at seven concentrations over three orders of magnitude, we demonstrate prediction of cytotoxicity in yeast from
\end{abstract}

Electronic supplementary material The online version of this article (doi:10.1007/s11306-011-0386-0) contains supplementary material, which is available to authorized users.

S. Heux $\cdot$ N. Zamboni $\cdot$ U. Sauer $(\bowtie)$

Institute of Molecular Systems Biology, ETH Zurich,

Wolfgang-Pauli Strasse 16, 8093 Zurich, Switzerland

e-mail: sauer@ethz.ch

S. Heux · T. J. Fuchs · J. Buhmann - N. Zamboni - U. Sauer

Competence Center for Systems Physiology and Metabolic

Diseases, Schafmattstrasse 18, 8093 Zurich, Switzerland

T. J. Fuchs · J. Buhmann

Department of Computer Science, ETH Zurich,

Universitätstrasse 6, 8092 Zurich, Switzerland intracellular metabolome patterns obtained at much lower drug concentrations that exert no physiological toxicity. Furthermore, the ${ }^{13} \mathrm{C}$-determined intracellular response of metabolic fluxes to drug treatment demonstrates the functional performance of the network to be rather robust, until growth was compromised. Thus we provide evidence that phenotypic robustness to drug challenges is achieved by a flexible make-up of the metabolome.

Keywords Yeast metabolism - Drug enzyme interaction . Off-target drug effects - Metabolomics - Method .

Machine-learning

\section{Introduction}

Predicting cellular behavior at the molecular level is an enormous challenge. One of the prerequisites for this endeavor is knowledge of the interactions between and responses to perturbations of the various molecules that make up cells. While our ability to interrogate gene-gene, protein-protein and protein-DNA interactions has greatly improved, methods to unravel metabolite-protein interactions or to quantify response to alterations in metabolite levels-both natural and artificial-are much less developed. The so far most successful approach is based on external supplementation of small molecules in so-called chemical genomics, most powerfully applied in combination with mutant libraries for systematic testing of conditionspecific genotype-phenotype associations (Hillenmeyer et al. 2008; Kapitzky et al. 2010; Lehar et al. 2008; Perlstein et al. 2007). While large numbers of combinatorial perturbations can be tested in parallel, typically only growth is quantified to identify functional interactions between added compound and modified gene. Slightly larger numbers of 
output variables can be screened through cell-based assays as exemplified by successful probing of four oxidative phosphorylation parameters (Wagner et al. 2008). Image-based assays can further expand the scope by extracting either individual optical features in the acquired images or more complex phenotypic traits from feature patterns (Perlman et al. 2004; Wagner and Clemons 2009; Young et al. 2008). In general, an inverse relationship exists between the extent of multiplexing and throughput of an assay (Feng et al. 2009). For primary screening of drug responses that requires high-throughput, the current practice is therefore targeted monitoring of up to a handful of cellular parameters or image-based detection of high level, multifactorial phenotypic traits.

In particular for drug development, however, richer data sets with tens to thousands of measured variables are necessary to address the key problems of target identification, mode of action, metabolic fate, biomarkers or toxicity. For many of these questions, metabolism is at the core of the matter because drug targets are often enzymes, enzymes convert drugs, drugs have metabolic consequences that might be exploitable as biomarkers, or because the drugs themselves or their metabolic products exhibit toxic effects. It is thus paramount to develop multi-variable assays that monitor the functional output of metabolism, which should be either at the level of fluxes or concentrations of metabolites (Coen et al. 2008; Ellis et al. 2007; Kaddurah-Daouk et al. 2008; Sauer 2004; Turner and Hellerstein 2005). A sufficiently high level of throughput is pivotal to exploit the potential of small-molecule phenotypic profiling in drug discovery and development (Wagner and Clemons 2009). In contrast to the powerful multi-variable output of transcriptomic or proteomic analyses that is simply neither feasible nor affordable on thousands of samples (Feng et al. 2009), mass spectrometry (MS)-based metabolomics (Zamboni and Sauer 2009) or fluxomics (Zamboni and Sauer 2004) are, in principle, capable of the required throughput. While fluxbased methods assess the integrated functional network output of all molecular regulation and catalytic interactions (Hellerstein 2003; Sauer 2006), metabolite-based methods can sensitively reveal local responses of metabolism to perturbations (Boer et al. 2010; Fendt et al. 2010a).

In pharmacological development, the two major sources of drug attrition are efficacy and toxicity (Hopkins 2008), where toxicity testing in the twenty-first century is considered to rely on high-throughput tests in cell lines and dose-response modeling (Andersen and Krewski 2009; Committee on Toxicity Testing and Assessment of Environmental Agents 2007). One of the gaps between this grand vision and the current practice is that almost all functional screens are done at a single, physiologically effective drug concentration and not over large dose ranges (Perlman et al. 2004). Here we attempt to fill this gap by developing a high throughput metabolomics method that enables prediction of cytotoxic drug effects by a machinelearning approach from a small number of assays at low drug dosages. As a proof of concept, we focused on central metabolism of the yeast Saccharomyces cerevisiae because potential cytotoxic effects of drugs can be expected to directly or indirectly affect this ubiquitous core network.

\section{Materials and methods}

\subsection{Strains, chemicals, and media}

Reagents (Supplementary Table 1) were purchased from Sigma Aldrich and stored at $-20^{\circ} \mathrm{C}$ until use. Each compound was dissolved in dimethyl sulfoxide (DMSO), with the exception of the water-dissolved drugs ara-CMP, caffeine, phenformin, doxorubicin, hydroxyurea, 2-deoxyglucose, oxythiamin, malonate and sodium iodoacetate. All experiments were performed with the $S$. cerevisiae strain FY4 MATa. Cultures were initially propagated in YPD plates and then grown in defined minimal medium (Verduyn et al. 1992) with $1 \%(w / v)$ glucose as the sole carbon source. The medium was buffered with $10 \mathrm{mM} \mathrm{KH-phthalate} \mathrm{(pH} \mathrm{5.0).}$

\subsection{Drug treatment}

To determine appropriate drug concentrations, a dilution series of each compound was made with either water or DMSO for five dilution steps, where the starting concentrations were chosen based on literature data. A $100 \mu$ l (for water-solved drugs) or $5 \mu \mathrm{l}$ for (for DMSO-solved drugs) aliquot of each dose or control (e.g. water or DMSO) was distributed to a 96 deep-well plate (Kühner AG, Switzerland) in triplicate in $1.2 \mathrm{ml}$ of minimal medium. From an overnight preculture, cells were added to each of the 96 wells to a final $\mathrm{OD}_{600}$ of 0.05 . Plates were then incubated at $30^{\circ} \mathrm{C}$ on a rotary shaker at $300 \mathrm{rpm}$ with $5 \mathrm{~cm}$ amplitude. The exponential growth phase of each culture was monitored by measuring the optical density at $600 \mathrm{~nm}\left(\mathrm{OD}_{600}\right)$ in a spectrophotometer (Molecular Devices, USA), and the maximal specific growth rate was determined by log-linear regression of $\mathrm{OD}_{600}$ versus time, with specific growth rate $\left(\mu_{\max }\right)$ as the regression coefficient. Based on these data, we chose seven concentrations for each drug that cover four orders of magnitude.

\subsection{Cultivation and metabolome sampling for shake flasks}

Yeast cultivations were performed in duplicate $500 \mathrm{ml}$ baffled shake flask $(250 \mathrm{rpm})$, at $30^{\circ} \mathrm{C}$ in minimal medium. Each flask contained $100 \mathrm{ml}$ of minimal medium. From 
biological triplicate experiments, $1 \mathrm{ml}$ aliquots of the midexponential growth phase at an $\mathrm{OD}_{600}$ of unity were rapidly withdrawn by pipetting, and quenched in $4 \mathrm{ml}-40^{\circ} \mathrm{C}$ precooled $60 \%$ (v/v) methanol with $10 \mathrm{mM}$ ammonium acetate at $\mathrm{pH}$ 7.5. Biomass was separated from the quenching solution by rapid centrifugation at $10,000 \mathrm{rpm}$ for $1 \mathrm{~min}$ at $-20^{\circ} \mathrm{C}$, and metabolites were extracted immediately by adding $1 \mathrm{ml} 80^{\circ} \mathrm{C} 75 \%(\mathrm{v} / \mathrm{v})$ ethanol containing $10 \mathrm{mM}$ ammonium acetate at $\mathrm{pH}$ 7.5. The mixture was incubated for 3 min at $80^{\circ} \mathrm{C}$ and then immediately chilled in an ice bath for $3 \mathrm{~min}$. Cell debris was separated by centrifugation at $10,000 \mathrm{rpm}$ for $10 \mathrm{~min}$ at $4^{\circ} \mathrm{C}$. The supernatant was dried at $30^{\circ} \mathrm{C}$ and 0.12 mbar using a Christ RVC2-33 concentrator vacuum system connected to Christ Alpha 2-4 LD plus freeze dryer (both from Kühner AG, Switzerland). After evaporation of the solvent, samples were stored at $-80^{\circ} \mathrm{C}$.

\subsection{Cultivation and metabolome sampling for filter plates}

To enable large scale metabolome analysis, batch growth experiments were carried out in 96-well plates with PES $0.45 \mu \mathrm{m}$ filter on the bottom (Interchim, France). Wells were filled with $1.2 \mathrm{ml}$ minimal medium, using four replicate wells per condition and 12 for the control. Inoculation was done with from overnight precultures to a final starting $\mathrm{OD}_{600}$ of 0.05 . Filter plates were incubated at $30^{\circ} \mathrm{C}$ using an orbital shaker with $5 \mathrm{~cm}$ amplitude at $300 \mathrm{rpm}$. To ensure appropriate mixing, one $4 \mathrm{~mm}$ diameter glass bead (Sigma-Aldrich, Switzerland) was added to each deep-well. Aliquots were withdrawn during the mid-exponential phase defined as an $\mathrm{OD}_{600}$ between 0.5 and 1.5). Supernatant was removed by vacuum-filtration through the well bottom within $30 \mathrm{~s}$. The filter plate with retained cell pellets was then quickly frozen with liquid nitrogen. Thus, quenching was achieved within $50 \mathrm{~s}$, and the results were consistent for most of the tested metabolite levels in control shake flask experiments with $15 \mathrm{~s}$ quenching (Supplementary Table 3). Metabolites were extracted from frozen pellets by adding $0.5 \mathrm{ml}$ of extraction solvent $(75 \%(\mathrm{v} / \mathrm{v})$ ethanol, $10 \mathrm{mM}$ ammonium acetate at $\mathrm{pH} 7.4$ ) at $-20^{\circ} \mathrm{C}$ to each well, followed by incubation in a water bath at $80^{\circ} \mathrm{C}$ for $3 \mathrm{~min}$. The liquid extracts were then collected into a 96-well collection plates by vacuum-filtration through the well bottom The pellets were washed once with $0.5 \mathrm{ml}$ of extraction solvent and the resulting extracts transferred in the same collection plate to yield ca. $1 \mathrm{ml}$ total extract. These were dried and stored as described above.

\subsection{High-throughput metabolomics}

The flow injection analysis was performed using an Agilent 1100 Series HPLC System coupled to an Applied
Biosystems/MDS SCIEX 4000 QTRAP instrument equipped with the TurboV electrospray interface. The samples from the evaporated plate were resuspended in $80 \mu \mathrm{l}$ distilled water and $5 \mu \mathrm{l}$ were transferred into a 96-well PCR plate (ABgene, UK) and then placed at $4^{\circ} \mathrm{C}$ in the autosampler. One microliter sample was injected in $75 \%(\mathrm{v} / \mathrm{v})$ methanol, $5 \mathrm{mM}$ ammonium carbonate at $\mathrm{pH} 9$ with a flow rate of $0.1 \mathrm{ml} / \mathrm{min}$. Spectra were collected in negative mode and in multiple reaction monitoring. A total of 68 transitions were acquired with $30 \mathrm{~ms}$ dwell time, and total cycle time of $2.3 \mathrm{~s}$. The routine throughput was of about 60 injections per hour, and was primarily set by the time necessary for cleaning of the injector and flushing of the ionization chamber to reduce carryover. The electrospray source was operated at $4.5 \mathrm{kV}$ and $650^{\circ} \mathrm{C}$. Compoundspecific settings were the same as in reference (Buscher et al. 2009). Data were recorded and analyzed with Analyst Software Version 1.4.2. For purposes of validation, extracts of yeast were prepared from 96-well filter plates as described. To assess linearity, we prepared a dilution series with such extracts and applied linear regression to the integrated peak areas for 68 compounds. To estimate intraand inter-day MS reproducibility, two different extracts from yeast cells grown in the same filter plate were split in two and stored at $-80^{\circ} \mathrm{C}$. The first fractions were analyzed four times on Day 1 and the remaining fractions were analyzed four times on Day 2. To approximate both interand intra- cultivation and sampling reproducibility, three different extracts from yeast cells grown in the same filter plate plus three different extracts from yeast grown in three different filter plates on three different days were all analyzed by flow injection-MS on the same day. For comparison, signals were normalized by dividing the peak area obtained for each metabolite with the $\mathrm{OD}_{600}$ of the culture.

\subsection{Metabolomics data processing}

For each sample, integrated metabolite areas were normalized with the corresponding OD at the time point of sampling and averaged over all replicates $(n=4$ for drugged cells, $n=12$ for controls). To ensure quality, metabolite concentrations for which either less than three replicas were measured or the relative error was larger than $35 \%$, were removed from the design matrix and indicated as "missing". The data was sorted by increasing drug concentration. Missing values were imputed linearly with the average of their closest neighbors with lower and higher concentration if both neighbors are measured. Metabolites which still comprised missing values were removed from the corresponding drug experiment. All metabolite areas were first normalized with the average area measured for the same metabolite in the (un-drugged) control and second by $\log 2$ transformation resulting in fold 
changes. Finally, metabolites were removed, which showed erratic behavior (i.e. single concentration spikes with a fold change of more than 0.6.).

For every profile of growth rates given by one drug at increasing concentration, we determined the maximum non-toxic concentration, which is the highest drug dose which does not affect the growth rate. This maximum nontoxic concentration is defined as the point $x_{i}$ in the growth rate profile for which $\left|x_{i+1}-\operatorname{avg}\left(\left\{x_{i+1}, x_{i}, x_{i-1}\right\}\right)\right|>0.02$.

\subsection{Toxicity prediction}

To potentially predict the occurrence of drug toxicity from metabolome measurements from the minimal number of sub-toxic concentrations, we attempted to train a general classifier for all drugs from the raw MS data. For each drug, we considered only drug concentrations lower or equal to the maximum non-toxic concentration, since predicting toxicity at a concentration at which toxicity is detectable at macroscopic scale is trivial. The design matrix $X$ for the classifier consists of metabolite concentration frames. A frame is defined as triplet of three consecutive drug concentrations, for each of which 68 non-filtered metabolite fold-changes were available and concatenated to form a single feature vector of length 204. These frames are overlapping, hence resulting in five frames for a drug, for which seven concentrations were measured and which showed no growth rate effect. For toxic drugs all doses above the maximum non-toxic concentration were neglected, and thus fewer frames could be extracted. The target variable $Y$ of the model is dependent on the growth rate profile of the drug. The frames were labeled based on the future onset of a growth rate effect of the drug, e.g. a frame consisting of concentrations two, three and four was labeled as "toxic" if the maximum nontoxic concentration was five or six. In the case of non-toxic drugs all five frames were labeled as "non-toxic".

One of the key challenges in this setting is the large number of missing values due to the rigorous quality control procedure. To overcome this problem we facilitated a k-nearest neighbor $(\mathrm{kNN})$ classifier which operates only on pairwise distances instead of using the whole design matrix at once (Hastie et al. 2009). The Euclidean distance was used to determine neighbors in the 204-dimensional feature space and $\mathrm{k}$ was set to 3 . To accommodate the fact that only one-third of all frames are flagged as toxic and the importance of high sensitivity in toxicity classification, a test sample was classified as "toxic" if one or more of its three nearest neighbors was "toxic". Prediction performance is measured with class balanced error rate. The main difficulty in learning a kNN classifier is feature selection, which aims at finding a subset of metabolites that yield the best performance in terms of toxicity prediction. The underlying hypothesis is that not all metabolite measurements are indicative for future toxicity and therefore contaminate the distance measure. In addition, since MS detection suffers from an intrinsic tradeoff between number of metabolites and precision, feature selection enables to prioritize MS detection and thus favorably affects data quality. To this end we implemented a forward-backward selection procedure, which optimizes the balanced error rate by adding and removing features until convergence (Hastie et al. 2009). The resulting subset is then used to predict the toxicity of the left out test samples. The learning procedure yields a toxicity model with a training error of $9 \%$.

We validated the classifier with two cross validation experiments. The first one was a leave one out cross validation on drug level. Hence all frames of a drug were left out of training and subsequently were predicted by the learned $\mathrm{kNN}$ model. This was repeated for each drug resulting in a prediction error of 24\% (Supplementary Fig. 6). The aim of the second validation experiment was to ensure that the whole training and selection procedure is not over fitting., To this end 100 permutation tests were conducted for leave-one-drug-out cross validation. At each run the target variable $Y$ was randomly permuted and the complete training and testing procedure was repeated with these permuted labels. The resulting average balanced error rate of $51 \%$ demonstrates that the learning procedure is not over fitting (Supplementary Fig. 6).

\section{$2.8{ }^{13} \mathrm{C}$ metabolic flux analysis}

Duplicate labeling experiments were carried out in 96 deepwell plate (Kühner AG, Switzerland) in $1.2 \mathrm{ml}$ of minimal medium on $10 \mathrm{~g} / \mathrm{l}$ glucose as a mixture of $80 \%(\mathrm{w} / \mathrm{w})$ naturally and $20 \%(\mathrm{w} / \mathrm{w})\left[\mathrm{U}_{-}{ }^{13} \mathrm{C}\right]$ labeled glucose $\left({ }^{13} \mathrm{C} \geq 99 \%\right.$, Cambridge Isotope Laboratories, USA). Each of the 41 compounds was administered at two concentrations as described in the main text. Cells from overnight cultures were harvested by centrifugation and washed using sugar free minimal medium to remove residual, unlabeled carbon source. Cultures were inoculated to an $\mathrm{OD}_{600}$ of 0.05 and harvested during mid-exponential growth $\left(\mathrm{OD}_{600} 0.5-1.2\right)$ by centrifugation. Residual medium was removed by washing the pellet three times with water and cells were stored at $-20^{\circ} \mathrm{C}$. Samples for gas chromatography-MS analysis were prepared as described previously (Blank et al. 2005) on a $6890 \mathrm{~N}$ instrument with a 5973 Inert XL MS system (Agilent Technologies). Flux ratios were determined from the mass isotopologue distribution of the proteinbound amino acids with the software FiatFlux (Zamboni et al. 2005) using the analytical equations developed by Fischer and Sauer (2003). The mass isotopologue distribution was corrected for the amount of unlabelled biomass and stable natural isotopes (Fischer and Sauer 2003). 


\section{Results and discussions}

\subsection{Physiological test of drug cytotoxicity in yeast}

To demonstrate characterization and prediction of druginduced toxicity at the functional level of metabolism, we assembled a set of 41 heterogeneous drugs (Supplementary Table 1). They were chosen to include (i) diverse mechanisms of toxic or therapeutic action in metabolic diseases or against fungal invasion; (ii) structurally diverse molecules with common targets; (iii) a wide range of metabolic targets; and (iv) some non-metabolic targets including signaling networks, transporters, DNA replication and cell division. Of the 34 drugs with known targets in yeast metabolism, ten are inhibitors of enzymes in central and amino acid metabolism. For each compound, we performed quantitative miniscale yeast growth experiments to assess its dose-dependent toxicity, defined here as a decrease in the specific growth rate. Based on this initial physiological characterization, we chose the concentration causing a 50\% reduction in growth rate as the highest dosage for our experiments. Starting from this drug-specific maximum concentration, we used seven sequentially lower drug concentrations that cover a range of four orders of magnitude (Supplementary Table 2). We thereby achieved at least four sub-toxic concentrations for all drugs (Fig. 1; Supplementary Fig. 1 for details). For 21 compounds, notably titration up to the maximum solubility did not cause any detectable growth rate effect at any concentrations.

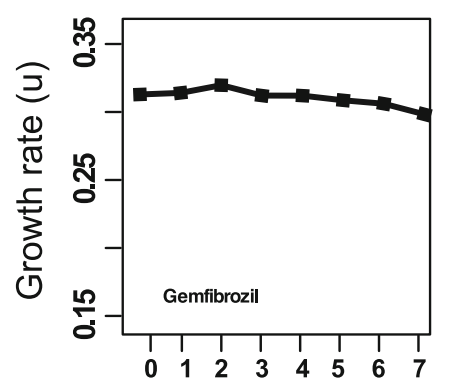

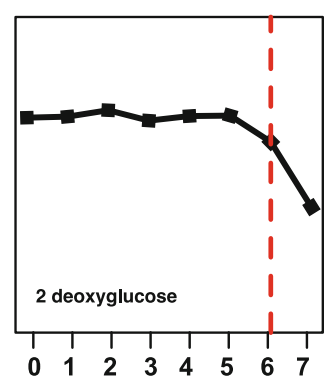

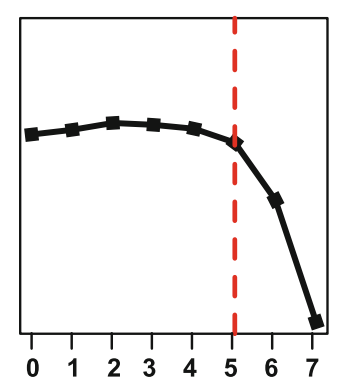

5-Fluorouacil

$\begin{array}{ll}\text { 6-Aminonicotinamide } & \text { 2-Deoxyglucose* } \\ \text { Bumetamide } & \text { Actinomycin D } \\ \text { Camptothecin } & \text { Ara-CMP } \\ \text { Chlopropamide } & \text { Fenpropimorph } \\ \text { Chlorothiazide } & \text { Malonate } \\ \text { Ciglitazone } & \text { Sulfometuron methyl } \\ \text { Fenofibrate } & \text { Tamoxifen }\end{array}$

Furosemide

Gemfibrozil* $^{*}$

Indapamide

lodoacetate

Lovastatin

Metformin

Nifedipine

Nimodipine

Orlistat

Oxythiamine

Repaglinide

Suramin

Tolbutamide

Troglitazone
Fig. 1 Yeast growth rate response to increasing drug concentrations. For each drug, eight drug concentrations were chosen to uniformly span over four orders of magnitude and such that the highest dosage (\#8) corresponds to the $\mathrm{IC}_{50}$ for yeast. Drugs were grouped by the onset of growth inhibition. Growth rates of one representative drug are shown (indicated by an asterisk). Vertical red lines indicate the highest non-toxic concentration. For absolute drug concentrations please consult Supplementary Table 2 
3.2 Assessing drug toxicity by ${ }^{13} \mathrm{C}$ metabolic flux analysis

Assuming that drugs are toxic because they inhibit essential pathways or perturb the overall energy and redox balance, we hypothesized that these effects could be reflected in changes of the intracellular reaction rates, triggered either passively by enzyme inhibition or actively by the cell to maintain homeostasis. Hence, we experimentally determined in vivo pathway activity by ${ }^{13} \mathrm{C}$-metabolic flux analysis (Sauer 2006; Zamboni et al. 2009) at two drug concentrations; i.e. the highest, often toxic concentration and the third lowest, always non-toxic concentration in the dilution series (Supplementary Table 2). The experiment consisted of growing yeast cultures on ${ }^{13} \mathrm{C}$-labeled glucose and computing seven independent ratios of converging intracellular pathways from the ${ }^{13} \mathrm{C}$-pattern recorded in proteinogenic amino acids by MS (Fischer and Sauer 2003; Sauer 2006; Zamboni et al. 2009). These ratios of converging fluxes provide a precise overview on carbon fluxes around key nodes within central metabolism. At the highest dosage, only nine drugs elicited minor flux changes that were probably an indirect consequence of altered physiology because the flux changes were rather similar
(Supplementary Fig. 2). More important, virtually no flux effect could be detected at the sub-toxic drug concentrations. Thus, metabolism is either impressively resilient to chemical perturbations or the employed flux method is not sufficiently sensitive to detect shifts that might occur in peripheral pathways. In either case, we concluded that such flux analysis is not pertinent for toxicity assessment.

\subsection{Development of a high-throughput metabolomics method}

As an alternative to flux analysis, we set out to investigate the metabolome response to drug treatment. In particular we wanted to exploit the recently demonstrated local sensitivity of metabolite concentrations to perturbations of enzyme activity, in particular when fluxes are invariant as can be expected when growth remains unaffected (Fendt et al. 2010a). To enable analysis of large sample cohorts in reasonable time, we established a high-throughput metabolomics platform for relative measurement of 50-100 metabolites in thousands of samples. The resulting workflow is depicted in Fig. 2a. Briefly, cultivation was performed in 96-well filter plates that enable comparable growth rates to shake flasks, and rapid quenching within
Fig. 2 High-throughput metabolomics workflow. a Analytical workflow. Yeast cells were grown in 96-well plates with filter bottom. For rapid quenching of metabolic activity, the medium was removed by suction and cell pellets on the filter were rapidly frozen in liquid nitrogen. Metabolites were extracted with buffered hot ethanol, and measured after flow injection on a triple quadrupole mass spectrometer at a rate of 1 sample/min. A total of 55 out of 68 targeted metabolites exhibited sufficient linearity and reproducibility for relative quantification. b Data processing workflow. An exemplary subset of six metabolite profiles is shown for a given drug. Raw data were first smoothed (red line) and noisy curves were filtered out. The earliest point of deflection from the control concentration (vertical green line) is detected as described in Sect. 2, and used to classify the profile (compare Fig. 1) a

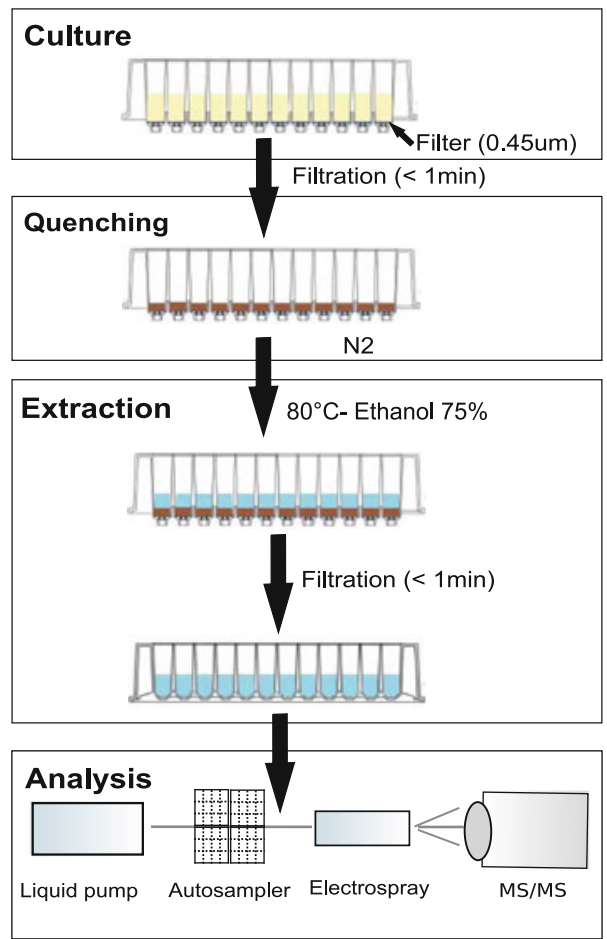

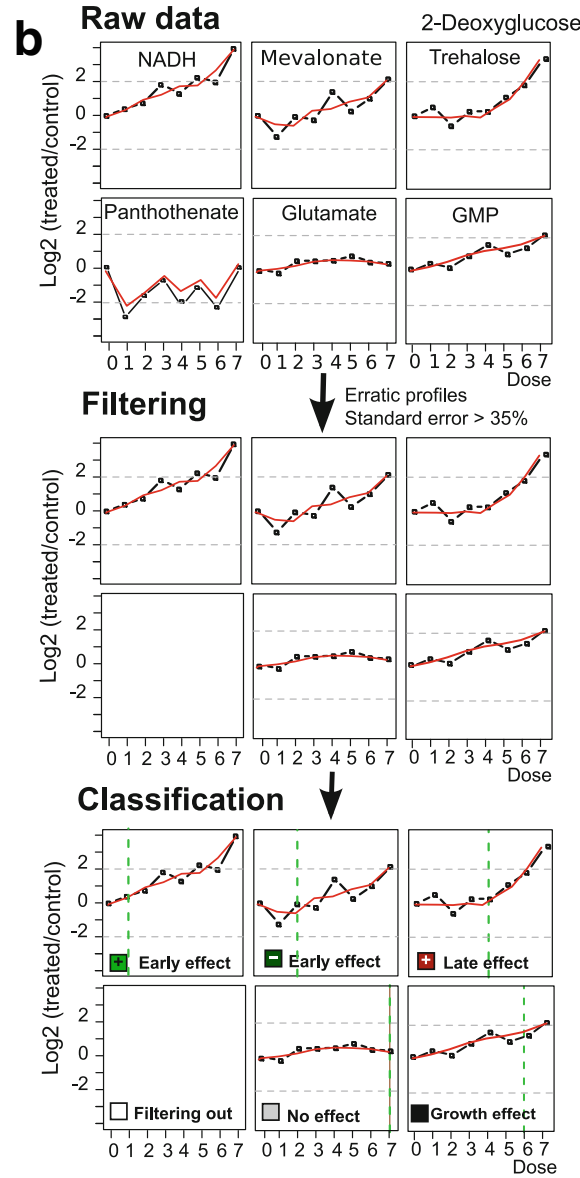


$50 \mathrm{~s}$ of biochemical activity was achieved by vacuum-filtering before solvent treatment. Intracellular metabolites were subsequently extracted using a standard procedure adapted to fit the here employed filter-based cell harvest, with a global recovery vs. standard protocol of typically above $75 \%$ (Supplementary Table 3). In total, about $10 \mathrm{~min}$ are required to process a plate with 96 cultures.

Cell extracts were analyzed by flow injection-tandem MS at a routine throughput of at least 1,200 samples/day. Mass spectrometer acquisition was initially devised to target 68 metabolites, corresponding to most intermediates of glycolysis, pentose-phosphate pathway, and the TCA cycle, as well as representatives of amino acids, nucleotides, polysaccharides, vitamins, and lipid precursors (Supplementary Table 4). From the original list of 68 intermediates, 13 failed quality control because of insufficient signal-to-noise, linearity, or reproducibility. For all of the 55 remaining metabolites, the mass spectrometer response was linear to the amount with $R^{2}>0.90$, and $R^{2}>0.95$ for more than 40 metabolites (Supplementary Table 5).

Before attempting a large screen, we tested all relevant reproducibility parameters. Intra-day and inter-day repeatability were found to have average relative standard deviations of $14 \%$ and $17 \%$, respectively (Supplementary Fig. 3). Similarly, sample-to-sample variability exhibited an average relative standard deviation of $19 \%$ within the same plate and $24 \%$ between plates, indicating that biological variability is larger than the technical precision. Further, noise and robustness were tested with yeast cells grown in the presence of compounds generally regarded to be inactive on yeast metabolism. As expected, most tested compounds did not affect the measurable metabolome. The only exception was xylose that cannot be utilized by yeast (Sonderegger and Sauer 2003), but surprisingly produced a specific metabolome pattern in its catabolic route (Supplementary Figs. 4, 5).

\subsection{Assessing drug cytotoxicity by high-throughput metabolomics}

To diagnose the effect of drugs on metabolism, we recorded metabolome profiles with our high-throughput platform from more than 1,500 samples, comprising 41 drugs at seven concentrations from four biological replicates plus controls. For each sample, 55 metabolites were measured, yielding more than 100,000 data points in total. To qualify drug effects, we generated dose-response curves that describe the relationship between metabolite changes and drug concentrations (Fig. 2b). Noisy signals and erratic profiles were neglected. For a one glance general overview of this large and multidimensional dataset, we condensed each metabolite-dose curve into a single value using an algorithm that identifies the lowest concentration at which a metabolite concentration deviates from its reference state in untreated cells. Thereby we can distinguish metabolites that are generally constant from those that exhibit a drugdependent trend. In particular, we were interested in identifying metabolites that respond already to sub-toxic drug concentration. Specifically, changes were defined as early if occurring within the three lowest concentrations tested, which correspond to doses lower than one tenth of the toxic concentrations that decrease growth rate (Fig. 2a).

In sharp contrast to the flux data, the high-throughput metabolome data revealed at least one early change at subtoxic dosage in $90 \%$ of the cases (i.e. 37 out of 41 drugs, Fig. 3). At least one late (i.e. at higher drug dosage) change was observed for 29 compounds, 17 of which also exhibited a growth defect. On average more than five early changes and two late changes were observed per drug before growth was affected, but there were striking differences between drugs. Specifically, 15 drugs exhibited less than five metabolite changes at sub-toxic dosages (cfr. Group A in Fig. 3), 14 drugs showed five to ten changes (cfr. Group B), and 12 drugs were characterized by more than ten changes (cfr. Group C). In the latter group, caffeine and suramin were two outliers with particularly widespread changes. While caffeine is known to inhibit proteins involved in the regulation of a wide variety of biological process (Reinke et al. 2006), suramin targets the DNA recombination machinery and thus affects genomic stability (Porcu and Chiarugi 2005; Schuetz et al. 2007). Group A with the fewest changes consists mostly of drugs from the therapeutic groups I, II and III that failed to impact growth even at the highest dosage. These drugs either lack a relevant target in yeast, are not transported, or elicited changes that are not detectable by screening responses in primary metabolism. For instance, fenofibrate binds to the peroxisome proliferator activated receptor alpha (Supplementary Table 1) that does not exist in yeast. Nimodipine and nifedipine target ion transporters that are likely to escape our screen because ions were not monitored. The highly diverse-changes group $\mathrm{C}$, in contrast, consists mostly of drugs that inhibit central, amino acids and nucleotide metabolism and, in turn, growth. Finally, drugs in the group B belong to various therapeutic groups (III, IV, V, VI and VII).

One key goal of such screening methods is drug target identification. For few drugs such primary target identification was in principle possible because the target was close to a detected metabolite. A good example is the pyruvate dehydrogenase inhibitor oxythiamine, whose supplementation caused only four metabolite responses, two of which were substrate and product of the reaction, pyruvate and acetyl-CoA. Another example is sulfometuron-methyl that inhibits the valine biosynthesis enzyme 


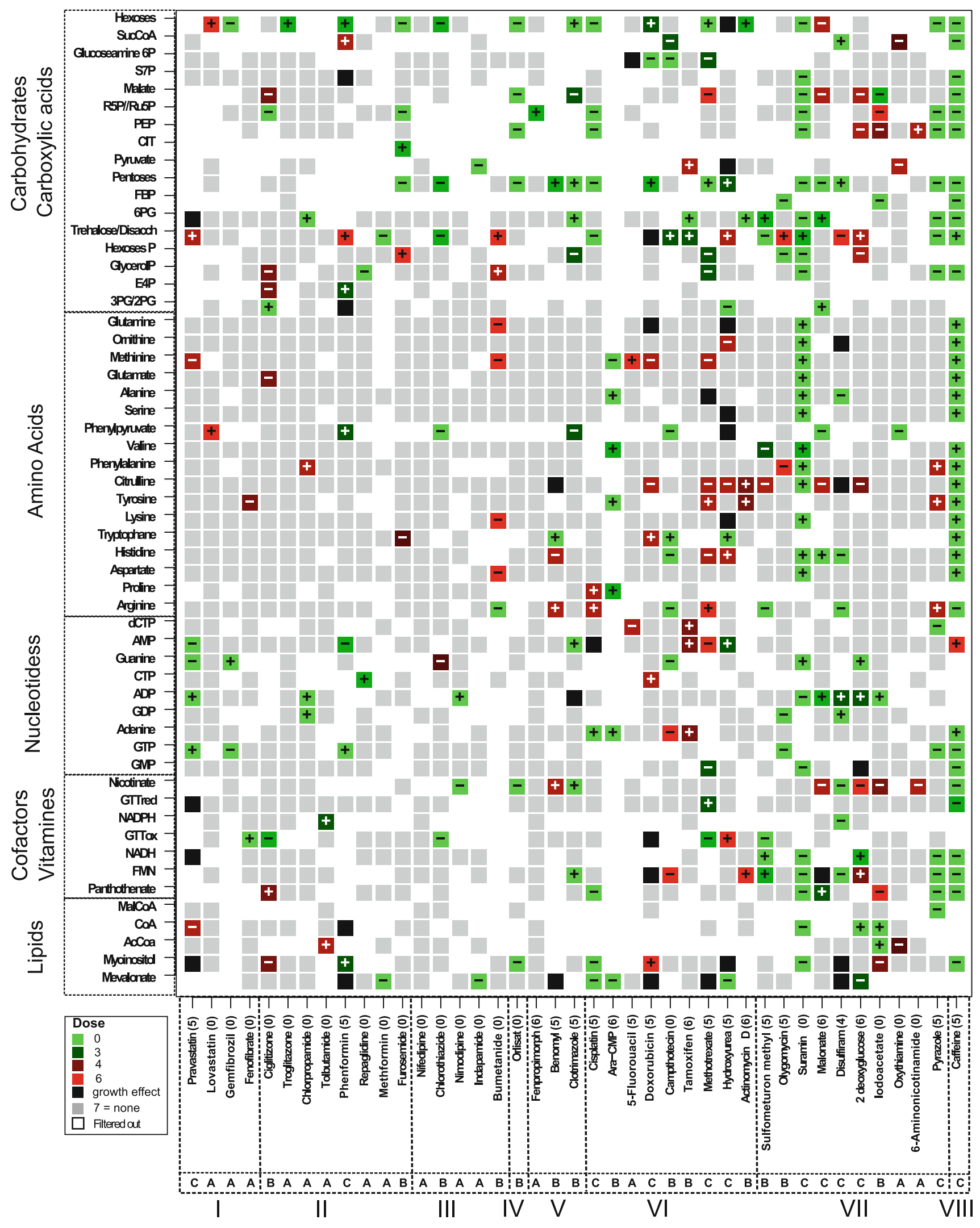


4Fig. 3 Metabolite profile effect map in response to drug treatment. The color code indicates at which drug concentration a metabolite response was detectable (according to the procedure shown in Fig. 2b), where green and red specify responses that occur at very low/medium and red at higher drug concentrations (see the bottom inset for definition). Increasing and decreasing metabolite concentrations are indicated by plus and minus, respectively. Black squares indicate metabolites that changed with or after growth decreases, gray squares indicate unaltered metabolites, and white squares highlight noisy metabolites that were filtered out. The precise drug dosage at which the physiological growth effect occurs is given in parentheses behind the drug names. The letter below the drug names indicates the number of metabolite changes that were induced by the drug; i.e. $A$ 0-4, B 5-10 and $C$ more than 10 metabolites. Drugs are classified according to their therapeutic area: group I: anti-hypercholesterolemia agents; group II: anti-diabetes agents; group III: anti-hypertension agents; group IV: anti-obesity agents; group V: antifungal; group VI: anti-cancers agents; group VII: inhibitors of the central carbohydrates metabolism; group VIII: stimulant

acetolactate synthase (Supplementary Table 1) and led to decreased concentration of the pathways product valine. The coverage of the presently employed metabolomics method, however, was not ideally suited to identify primary drug targets outside of primary metabolism.

\subsection{Early cytotoxicity prediction from metabolome profiles}

Surprisingly, several metabolite shifts were reproducibly detected at drug levels well below the highest non-toxic concentration (cfr. green squares in Fig. 3). These early changes in metabolites were not necessarily in the immediate vicinity of the known target. For example, sugar levels often increase upon drug insult (cfr. hexoses, pentoses, and disaccharides in Fig. 3), indicating a mobilization or a production of storage carbohydrates upon a general stress (Wiemken 1990). The existence of conserved early changes in metabolome profiles in response to nontoxic drug treatment potentially enables to predict toxicity before it is physiologically manifested in the growth rate.

For this purpose, we resorted to machine learning to train a classifier. Specifically, we attempted to predict cytotoxicity from the trajectories of metabolites measured at three consequent sub-toxic drug concentrations. Technically, toxicity is defined as a drop in the growth rate, which results in a kink in the growth rate profile. Importantly, we set out to use raw data and only removed single data points that were not reproducible, thus bypassing the filtering procedure (Fig. 2b) that requires the measurement of full drug-response profiles. To cope with gaps in the data set, we trained a 3-nearest neighbor classifier operating at pair-wise distances, and applied a forward-backward selection procedure to filter uninformative features. The training procedure identified ornithine, glutamate, citrulline, $\alpha$-ketoglutarate, and fructose-bis-phosphate as the most informative metabolites for toxicity prediction on all drugs. The classifier performed well on the training dataset (Fig. 4). In general, the classifier successfully predicts drug toxicity (i.e. reduced growth rate) from rather low sub-effective concentrations of most tested drugs. Sporadically, misclassifications occur in gemfibrozile, iodoacetate, bumetamide, caffeine, fenpropimorph, and orlistat. Only in the case of suramin, the classifier consistently fails to correctly interpret the metabolome profiles because the metabolites vary strongly despite the absence of a detectable growth defect. It is possible, however, that toxic effects indeed occur at the next higher, not tested concentration. We validated the classifier with two cross validation experiments, and performed permutations tests to check for overfitting (Supplementary Fig. 6). Based on these tests we conclude that a balanced error rate of $24 \%$ is a well-justified estimate of the generalization error of the proposed classification model. Furthermore, the cross validation experiments showed only a marginal higher sensitivity than specificity for cytotoxicity (Supplementary Table 6).

\section{Concluding remarks}

Overall, we conclude that independent of the drug class, the specific mechanism of action, or the absolute drug concentration, the classifier correctly predicts growth defects that occur at higher dosage from metabolome trajectories in three quarters of the cases. This also suggests the existence of metabolome pattern that are characteristic of a stress response at lower drug concentrations that precede physiological cytotoxicity. These results demonstrate that the composition of the metabolic network in terms of its metabolite concentrations is very sensitive to drug treatment, while the functional network output in terms of metabolic fluxes is rather robust, until growth is compromised. This robustness of the fluxome response is consistent with findings using genetic perturbations (Fendt et al. 2010b; Fischer and Sauer 2005). The sensitive metabolome response, in contrast, renders metabolomics a very promising methodology for biomarker and toxicity screening. As demonstrated here with more than thousand samples analyzed per day on one instrument, metabolomics eclipses flux and other omics methods by at least one order of magnitude in terms of throughput and cost. The presented yeast cytotoxicity data cannot be directly translated to humans, as illustrated by the apparent absence of growth and metabolome effects of troglitazone-yet the drug was removed from the market for high incidents of liver damage. Reasons include the absence of many drug targets in yeast (Supplementary Table 1) and the fact that many of 


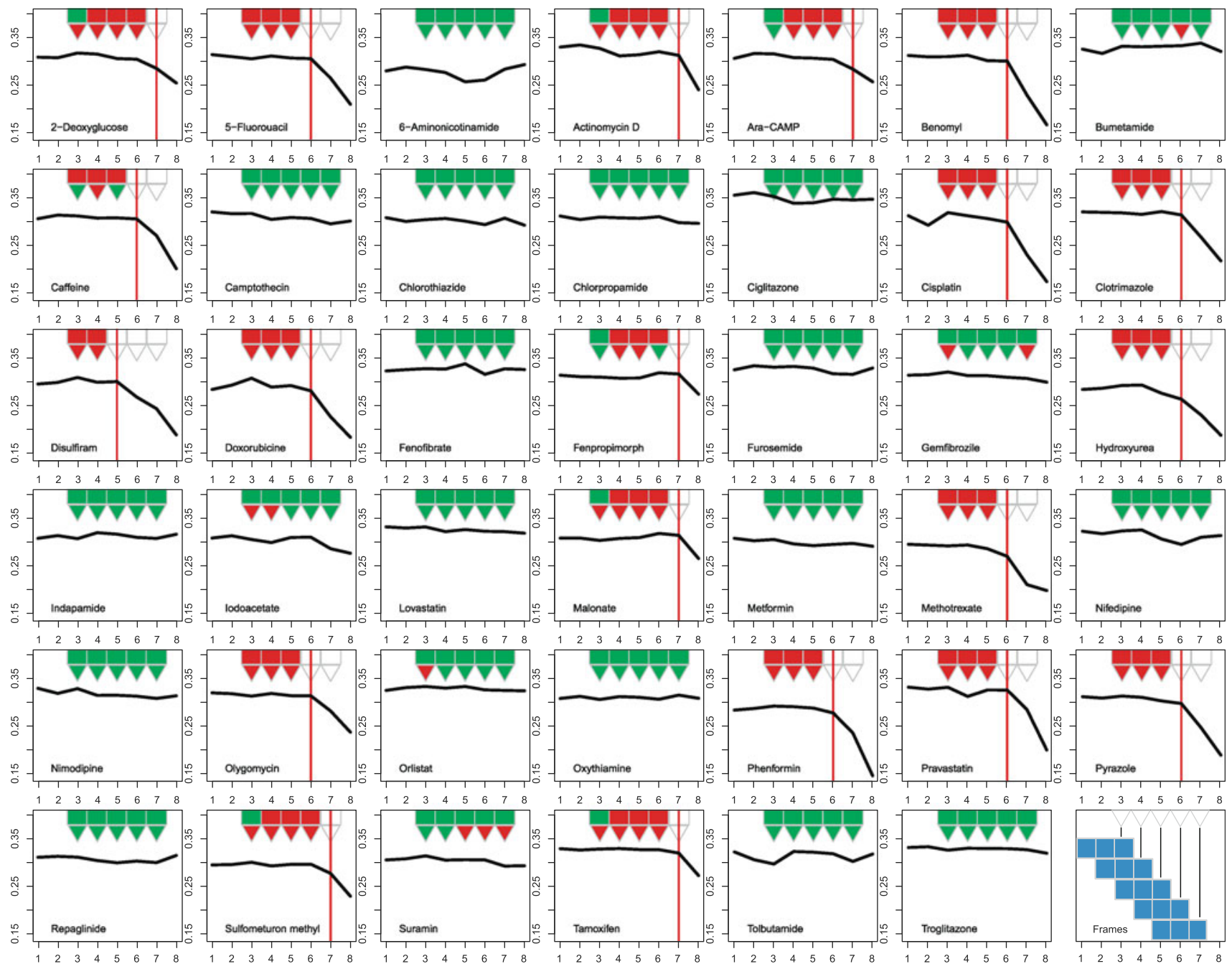

Fig. 4 Prediction of drug toxicity based on intracellular metabolite profiles. Each plot reports the dose dependent growth rate (black line) and onset of toxicity (vertical red line) for each drug. For all frames of three consecutive drug dosages (visualized in the bottom right plot), we applied the previously trained toxicity classifier on the metabolite profiles to predict whether the drug will affect growth at a higher dose. Red and green rectangles symbolize the ground truth labeling, while triangles visualize the prediction of the classifier for the given frame. Red indicates that the drug is going to be toxic at a higher dose. Green indicates that the drug is predicted to be non-toxic at higher dose. All points above the maximum non-toxic concentrations were excluded from training and testing. An example of a misclassified frame is bumetamide the mammalian drug-metabolizing enzymes are not present in yeast. The presented approach to rapidly detect or anticipate cytotoxic side effects, however, is generally applicable beyond microbes also to higher cells. Obviously, human cytotoxicity should be investigated in human cell lines to which the sample processing workflow can be adapted. More importantly, the prediction power can be significantly increased by using high-resolution mass spectrometers to resolve hundreds of metabolites, possibly in a dynamic fashion.

Acknowledgments S.H. gratefully acknowledges funding by Marie Curie Intra-European Fellowships (F6P-2005).

\section{References}

Andersen, M. E., \& Krewski, D. (2009). Toxicity testing in the 21 st century: Bringing the vision to life. Toxicological Sciences: An Official Journal of the Society of Toxicology, 107(2), 324-330.

Blank, L. M., Kuepfer, L., \& Sauer, U. (2005). Large-scale 13C-flux analysis reveals mechanistic principles of metabolic network robustness to null mutations in yeast. Genome Biology, 6(6), R49.

Boer, V. M., Crutchfield, C. A., Bradley, P. H., Botstein, D., \& Rabinowitz, J. D. (2010). Growth-limiting intracellular metabolites in yeast growing under diverse nutrient limitations. Molecular Biology of the Cell, 21(1), 198-211.

Buscher, J. M., Czernik, D., Ewald, J. C., Sauer, U., \& Zamboni, N. (2009). Cross-platform comparison of methods for quantitative 
metabolomics of primary metabolism. Analytical Chemistry, 81(6), 2135-2143.

Coen, M., Holmes, E., Lindon, J. C., \& Nicholson, J. K. (2008). NMR-based metabolic profiling and metabonomic approaches to problems in molecular toxicology. Chemical Research in Toxicology, 21(1), 9-27.

Committee on Toxicity Testing and Assessment of Environmental Agents, N. R. C. (2007). Toxicity Testing in the 21st Century: A vision and a strategy. Washington, DC: The National Academies Press.

Ellis, D. I., Dunn, W. B., Griffin, J. L., Allwood, J. W., \& Goodacre, R. (2007). Metabolic fingerprinting as a diagnostic tool. Pharmacogenomics, 8(9), 1243-1266.

Fendt, S. M., Buescher, J. M., Rudroff, F., Picotti, P., Zamboni, N., \& Sauer, U. (2010a). Tradeoff between enzyme and metabolite efficiency maintains metabolic homeostasis upon perturbations in enzyme capacity. Molecular Systems Biology, 6, 356.

Fendt, S. M., Oliveira, A. P., Christen, S., Picotti, P., Dechant, R. C., \& Sauer, U. (2010b). Unraveling condition-dependent networks of transcription factors that control metabolic pathway activity in yeast. Molecular Systems Biology, 6, 432.

Feng, Y., Mitchison, T. J., Bender, A., Young, D. W., \& Tallarico, J. A. (2009). Multi-parameter phenotypic profiling: Using cellular effects to characterize small-molecule compounds. Nature Reviews Drug Discovery, 8(7), 567-578.

Fischer, E., \& Sauer, U. (2003). Metabolic flux profiling of Escherichia coli mutants in central carbon metabolism using GC-MS. European Journal of Biochemistry, 270(5), 880-891.

Fischer, E., \& Sauer, U. (2005). Large-scale in vivo flux analysis shows rigidity and suboptimal performance of Bacillus subtilis metabolism. Nature Genetics, 37(6), 636-640.

Hastie, T., Tibshirani, R., \& Friedman, J. (2009). The elements of statistical learning. New York: Springer.

Hellerstein, M. K. (2003). In vivo measurement of fluxes through metabolic pathways: The missing link in functional genomics and pharmaceutical research. Annual Review of Nutrition, 23, 379-402.

Hillenmeyer, M. E., Fung, E., Wildenhain, J., Pierce, S. E., Hoon, S., Lee, W., et al. (2008). The chemical genomic portrait of yeast: Uncovering a phenotype for all genes. Science, 320(5874), 362-365.

Hopkins, A. L. (2008). Network pharmacology: The next paradigm in drug discovery. Nature Chemical Biology, 4(11), 682-690.

Kaddurah-Daouk, R., Kristal, B. S., \& Weinshilboum, R. M. (2008). Metabolomics: A global biochemical approach to drug response and disease. Annual Review of Pharmacology and Toxicology, $48,653-683$

Kapitzky, L., Beltrao, P., Berens, T. J., Gassner, N., Zhou, C., Wuster, A., et al. (2010). Cross-species chemogenomic profiling reveals evolutionarily conserved drug mode of action. Molecular Systems Biology, 6, 451.

Lehar, J., Stockwell, B. R., Giaever, G., \& Nislow, C. (2008). Combination chemical genetics. Nature Chemical Biology, 4(11), 674-681.

Perlman, Z. E., Slack, M. D., Feng, Y., Mitchison, T. J., Wu, L. F., \& Altschuler, S. J. (2004). Multidimensional drug profiling by automated microscopy. Science, 306(5699), 1194-1198.
Perlstein, E. O., Ruderfer, D. M., Roberts, D. C., Schreiber, S. L., \& Kruglyak, L. (2007). Genetic basis of individual differences in the response to small-molecule drugs in yeast. Nature Genetics, 39(4), 496-502.

Porcu, M., \& Chiarugi, A. (2005). The emerging therapeutic potential of sirtuin-interacting drugs: From cell death to lifespan extension. Trends in Pharmacological Sciences, 26(2), 94-103.

Reinke, A., Chen, J. C., Aronova, S., \& Powers, T. (2006). Caffeine targets TOR complex I and provides evidence for a regulatory link between the FRB and kinase domains of Tor1p. Journal of Biological Chemistry, 281(42), 31616-31626.

Sauer, U. (2004). High-throughput phenomics: Experimental methods for mapping fluxomes. Current Opinion in Biotechnology, 15(1), $58-63$.

Sauer, U. (2006). Metabolic networks in motion: 13C-based flux analysis. Molecular Systems Biology, 2, 62.

Schuetz, A., Min, J., Antoshenko, T., Wang, C. L., Allali-Hassani, A., Dong, A., et al. (2007). Structural basis of inhibition of the human NAD+-dependent deacetylase SIRT5 by suramin. Structure, 15(3), 377-389.

Sonderegger, M., \& Sauer, U. (2003). Evolutionary engineering of Saccharomyces cerevisiae for anaerobic growth on xylose. Applied and Environmental Microbiology, 69(4), 1990-1998.

Turner, S. M., \& Hellerstein, M. K. (2005). Emerging applications of kinetic biomarkers in preclinical and clinical drug development. Current Opinion in Drug Discovery \& Development, 8(1), $115-126$

Verduyn, C., Postma, E., Scheffers, W. A., \& Van Dijken, J. P. (1992). Effect of benzoic acid on metabolic fluxes in yeasts: A continuous-culture study on the regulation of respiration and alcoholic fermentation. Yeast, 8(7), 501-517.

Wagner, B. K., \& Clemons, P. A. (2009). Connecting synthetic chemistry decisions to cell and genome biology using smallmolecule phenotypic profiling. Current Opinion in Chemical Biology, 13(5-6), 539-548.

Wagner, B. K., Kitami, T., Gilbert, T. J., Peck, D., Ramanathan, A., Schreiber, S. L., et al. (2008). Large-scale chemical dissection of mitochondrial function. Nature Biotechnology, 26(3), 343-351.

Wiemken, A. (1990). Trehalose in yeast, stress protectant rather than reserve carbohydrate. Antonie Van Leeuwenhoek, 58(3), 209-217.

Young, D. W., Bender, A., Hoyt, J., McWhinnie, E., Chirn, G. W., Tao, C. Y., et al. (2008). Integrating high-content screening and ligand-target prediction to identify mechanism of action. Nature Chemical Biology, 4(1), 59-68.

Zamboni, N., Fendt, S. M., Ruhl, M., \& Sauer, U. (2009). (13)C-based metabolic flux analysis. Nature Protocols, 4(6), 878-892.

Zamboni, N., Fischer, E., \& Sauer, U. (2005). FiatFlux-a software for metabolic flux analysis from $13 \mathrm{C}$-glucose experiments. BMC Bioinformatics, 6, 209.

Zamboni, N., \& Sauer, U. (2004). Model-independent fluxome profiling from $2 \mathrm{H}$ and $13 \mathrm{C}$ experiments for metabolic variant discrimination. Genome Biology, 5(12), R99.

Zamboni, N., \& Sauer, U. (2009). Novel biological insights through metabolomics and 13C-flux analysis. Current Opinion in Microbiology, 12(5), 553-558. 